\title{
The role of Eutrema salsugineum cold shock domain protein EsCSDP3 in the cold-acclimation
}

Shamustakimova A.O.

All-Russia Research Institute of Agricultural Biotechnology, Moscow, Russia

e-mail:nastja_sham@mail.ru

Plant cold shock domain proteins (CSDPs) are DNA/RNA-binding proteins. CSDPs contain the conserved cold shock domain (CSD) in the N-terminal part and glycine-rich regions with varying number of the retroviral-like zinc fingers in the C-terminus. At present, the number of studies suggest an involvement of CSDPs in adaptation to the abiotic stress and the role in the plant growth and development.

The primary goal of this study is to understand the role of RNA-binding protein EsCSDP3 from Eutrema salsugineum in the cold-acclimation.

Our previous study have indicated that overexpression of EsCSDP3 increase tolerance to freezing in transgenic Arabidopsis thaliana.

RNA pull-down assay was conducted on A. thaliana with stable expression of EsCSDP3Halotag or Halotag only. Initial analysis determined that chimeric protein EsCSDP3Halotag saves ability to bind RNA with high affinity.

Rosette leaves from plants cultivated at $23{ }^{\circ} \mathrm{C}$ and then transferred to $4{ }^{\circ} \mathrm{C}$ for 3 weeks were ground in liquid nitrogen. Whole cell extracts containing the Halo tagged bait protein were incubated with affinity magnetic beads for $2 \mathrm{~h}$ at $4{ }^{\circ} \mathrm{C}$. The beads were washed with lysis buffer. Bound RNA-protein complexes were eluted by incubating the beads with buffer containing TEV protease. Total RNA was prepared from either whole cell extract or from the Halo purified samples using ExtractRNA reagent.

Comparative RNA profile were analyzed by Bioanalyzer 2100 using reagents for total and small/microRNA. RNA was reverse transcribed and the resulting cDNA was analyzed by PCR followed by agarose gel electroforesis. 